\title{
Risk Behaviours among Women in Artisanal Mining of Mineral Substances on Sites in Kolwezi, DRC
}

\author{
Comportements à risque chez les femmes en activités d'exploitation artisanale des substances minérales dans les
} sites miniers à Kolwezi, RDC

\author{
Hervé Mutombu Kabwit ${ }^{a^{*}}$, Clarence Mukeng a Kaut ${ }^{d}$, Kakoma Tshikishi Basile ${ }^{a}$ Hitshika Tshikolasonyi Papy ${ }^{a}$, Henri \\ Mundongo Tshamba ${ }^{a, b}$, Lukumwena Kalala Zet ${ }^{c}$, Raphael Bushabu Piema ${ }^{e}$ and Françoise Malonga Kaj \\ anstitut Supérieur des Techniques Médicales-Kolwezi, Kolwezi, République Démocratique du Congo; \\ bUniversité de Kolwezi, Faculté de Médecine, Kolwezi, République Démocratique du Congo; \\ 'Université de Lubumbashi, Faculté de médecine vétérinaire, Lubumbashi, République Démocratique du Congo ; \\ dUniversité de Kolwezi, Faculté de Médecine, Kolwezi République Démocratique du Congo ; \\ eUniversité de Lubumbashi, Faculté de Sciences Politiques et Administrative, département de sociologie, Lubumbashi, République Démocratique du \\ Congo.
}

Received 06 Nov 2017, Accepted 07 Jan 2018, Available online 12 Jan 2018, Vol.6 (Jan/Feb 2018 issue)

\begin{abstract}
Artisanal mining is currently part of the main mining activities in DRC. However, it's marked by problems such as the exploitation of vulnerable people in this case women and children. This study is carried out with the aim of identifying risky behaviour among women in the exercise of their activities within artisanal mine in Kolwezi. It is a cross-sectional descriptive study of 374 women working in the 11 artisanal mining sites between January 2017 and May 2017. The data was analyzed using software Epi Info 7.2.1.0. The most prevalent risk behaviours observed were lack of wearing PPE 93,32\% of the surveys, the use of mine water to ensure cleanliness of hands and utensils by $74,94 \%$ of inquiries, the seated position on the ores during grinding and sorting operations by $68,18 \%$ of surveys. These behaviours expose these women to the risks of irradiation and intoxication, given the levels of radiotoxic substances in these ores, which can be revealed somatically by disorders of several types.
\end{abstract}

Keywords: risk behaviours, women, artisanal mining, mineral substances, Kolwezi, DRC

L'exploitation minière artisanale fait partie actuellement de principales activités minières en RDC. Cependant, elle est marquée par des problèmes tels que l'exploitation des populations vulnérables en occurrence les femmes et les enfants. Cette étude est réalisée dans le but d'identifier les comportements à risque chez les femmes dans l'exercice de leurs activités au sein de la mine artisanale à Kolwezi. Il s'agit d'une étude descriptive transversale auprès de 374 femmes travaillant dans 11 sites d'exploitation minière artisanale entre Janvier 2017 et Mai 2017. Les données ont été analysées grâce au logiciel Epi Info 7.2.1.0. Les comportements à risques les plus prévalents observés sont l'absence de port des EPI pour 93,32\% des enquêtées; I'utilisation des eaux d'exhaure pour assurer la propreté des mains et des ustensiles par 75,94\% d'enquêtées; la station assise sur les minerais lors des opérations de broyage et de triage par 68,18\% d'enquêtées. Ces comportements exposent ces femmes aux risques d'irradiation et d'intoxication étant donné les teneurs des substances radiotoxiques dans ces minerais, qui peuvent se révéler au niveau somatique par des troubles de plusieurs natures.

Mots clés : comportement à risque, femmes, exploitation minière artisanale, substance minérale, Kolwezi, RDC

\section{Introduction}

Il est admis aux jours d'aujourd'hui qu'environ $30 \%$ des artisanaux du monde sont des femmes; et qu'elles occupent un certain nombre de rôles allant des

*Corresponding author's ORCID ID: 0000-0001-5811-6581

DOI: https://doi.org/10.14741/ijmcr.v6i01.10908 méthodes d'extractions jusqu'à la transformation [1]. Les données en rapport avec l'implication de la femme dans les activités minières artisanales montrent des fluctuations à travers le monde [2,3].

Les auteurs indiquent qu'en Asie, moins de $10 \%$ des mineurs sont des femmes, alors qu'en Amérique latine, cette proportion tend à être plus élevée, environ 10 à 64 |Int. J. of Multidisciplinary and Current research, Vol.6 (Jan/Feb 2018) 
$20 \%$. Ce pourcentage est plus élevé en Afrique, où il est compris entre 40 et $50 \%$ [4]. Au Mali, ce pourcentage est évalué entre 60 et $70 \%$ [5], tandis qu'au Ghana dans le district de Birmin ce pourcentage est estimé à $50 \%$ [6].

La RDC, pays à potentiel minier avec au moins 1000 différentes substances minérales [7], ainsi parle-t-on d'un scandale géologique, le secteur de la mine artisanale est le plus important de l'activité minière, dans la mesure où il présente le plus d'impact en termes de production économique et du nombre des personnes impliquées. Dans ce même pays, $66 \%$ de la population active est représentée par les femmes [8]. C'est dans ce contexte que travailler dans les sites d'exploitation artisanale des diverses ressources naturelles, dont le pays dispose en grande quantité, est l'une des activités de prédilection et de survie pour un grand nombre de femmes et des personnes ayant manqué de l'emploi dans le secteur industriel privé [8]. Les données se rapportant à leur implication dans ce secteur sont divergents en RDC. Certains auteurs estiment la présence féminine dans les sites miniers entre 16 et $20 \%[8,9]$, d'autres l'évaluent à $50 \%$ [1].

En dépit de l'implication croissante des femmes dans ce secteur, leur travail est souvent qualifié à tort de léger. Cette acception donne la fausse impression qu'il comporte peu ou pas des risques. Par ailleurs, cette acception se justifie dans la mesure où de nombreuses études ont décrit le caractère masculin de l'exploitation minière artisanale, peu des chercheurs ont étudié le rôle de la femme dans ce secteur [6] et les comportements à risque inhérent à leurs activités dans ce domaine. Et en $\mathrm{RDC}$, en dépit du caractère radiotoxique des minerais qui font l'objet de cette exploitation [10, 11, 12]. Cette étude vient pallier à cette insuffisance en montrant les comportements à risque observés chez les femmes dans l'exercice de leurs activités au sein des mines artisanales dans l'hinterland minier de la ville de Kolwezi et ses alentours.

\section{Méthodologie}

Cette recherche s'est effectuée dans la ville de Kolwezi, dans la province du Lualaba et dans sa partie Sud-Est, en RDC. Cette ville doit son existence à l'exploitation du cuivre et du cobalt par l'entreprise minière appelée la Générale des Carrières et des Mines (GECAMINES). Sa naissance remonte vers les années 1938. Elle est bornée au Nord par le territoire de Lubudi, au Sud-Est par la République de la Zambie et le district du Haut-Katanga, à l'Ouest par le territoire de Mutshatsha. Le sol est argilosablonneux. Le sous-sol est riche en gisements miniers du cuivre, de cobalt, de l'or, d'uranium et de radium [10, 11, 12 , 13]. Les activités économiques importantes sont industrielles et minières exercées par un certain nombre d'entreprises ainsi que les artisanaux [13]

La nature de cette étude nous a guidé à une enquête descriptive transversale qui nous a permis d'observer le comportement à risque durant les activités exercées par les femmes dans les sites d'exploitation artisanale des minerais. Il s'agit donc d'une enquête évaluant le comportement, l'attitude et les pratiques (CAP) des femmes travaillant dans ce secteur. La collecte des données a été prospective et a duré 5 mois, de janvier 2017 à Mai 2017. La population source a été constituée des femmes trouvées dans les sites d'exploitation artisanale de substances minérales au moment de notre enquête. La taille de l'échantillon a été calculée selon la formule $n \geq\left(Z_{\alpha}+Z_{\beta}\right)^{2} p q / e^{2}$ où $n=$ la taille de l'échantillon, $Z_{\alpha}=$ coefficient de confiance ou la valeur de $Z_{1-\alpha / 2}$ pour un test bilatéral ici 1,$96 ; Z_{\beta}=$ valeur de $Z$ pour une puissance $1-\beta$, nous avons souhaité une puissance de $90 \%$ qui équivaut à une valeur $1-\beta$ de 1,$282 ; p=$ proportion théorique des femmes fréquentant les mines artisanales, nous l'avons estimé à $5 \%$; q=proportion théorique de la population ne fréquentant pas les mines artisanales ( $q=1-$ p) ici $1-0,05=0,95$; e=erreur type requise, nous avons souhaité un niveau de confiance de $95 \%$.

L'application de cette formule abouti à l'échantillon suivant: $n \geq(1,96+1,282)^{2} 0,05 \quad(1-0,95) / 0,05^{2}=199,7$ femmes. A cette taille de l'échantillon, nous avons ajouté le nombre de non répondants que nous avons estimés à $20 \%$ en appliquant le coefficient de correction $C=1 / 1-f=$ $1 / 1-0,2=1,25$ où $C=$ coefficient de correction $; f=$ proportion d'erreur. La taille de notre échantillon était donc de $n^{*} c=200 * 1,25=249,6$ environ 250 femmes Pour augmenter la puissance statistique et diminuer l'erreur d'échantillonnage, nous avons porté notre échantillon à 374 femmes qui ont été, interviewées dans 11 sites d'exploitation minière artisanale. La sélection de ces sites a été réalisée de manière randomisée, chaque site a été considéré comme une grappe, au sein de quel, toute ouvrière trouvée ayant satisfait au critère, selon lequel être présente et âgée de plus de 12 ans au moment de l'enquête a été interviewée après avoir sollicité son consentement libre et éclairé.

Les variables d'intérêts ont été regroupées en variables sociodémographiques (âge, état civil, niveau d'instruction, état matrimonial, durée dans l'activité et le nombre d'heures réalisées dans le site par jour) ; les types d'activités réalisées par la femme. Les variables évaluant les comportements à risque ont été le port des équipements de protection individuel (EPI), la station assise sur les minerais au moment des opérations de broyage et de triage, l'entreposage des minerais dans la maison; l'utilisation des eaux d'exhaure pour assurer la propreté des certaines parties du corps tel que les mains, les pieds, le visage. Les données ont été saisies avec le logiciel Epi Info 7.2.1.0. Le logiciel Excel 2013 et Word 2013 nous ont aidés respectivement à présenter les tableaux et à traiter le texte.

Par ailleurs les ouvrières qui répondaient aux critères, leur consentement libre, éclairé a été recueilli avant l'interview. Les informations ont été obtenues dans la langue parlée de la participante, ou dans une langue comprise par elle, de façon standardisée et adaptée. La confidentialité des réponses a été garantie par l'équipe 
d'enquête, les entretiens se sont déroulés par ailleurs sans la présence de tierces personnes.

\section{Résultats}

Selon qu'il s'agit des sites où les enquêtées ont été retrouvées, nous avons constaté que $14,71 \%$ des femmes ont été rencontrées dans le site de Kisankala; le site de Kalumbwe a été le moins représenté avec $3,48 \%$ des enquêtées par rapport à l'ensemble des sites visités. La figure ci-dessous montre la répartition des femmes selon les sites où elles ont été retrouvées.

Lorsqu'on considère les caractéristiques sociodémographiques, notre échantillon était composé de $50,0 \%$ des femmes dont le niveau d'étude était primaire ; $81,02 \%$ étaient mariées ; $62,06 \%$ d'entre elles étaient épouses aux creuseurs ; 47,06\% résidaient dans les quartiers ou les villages situés à proximité des sites d'exploitation minière artisanale ; $43,85 \%$ avaient l'âge compris entre 20 et 30 ans, l'âge minimal a été de 13 ans l'âge maximal a été de 60 ans, l'âge moyen a été de 29,9 $\pm 9,9$ ans.

La durée moyenne dans cette activité a été de 3,3 32,3 ans. Elles réalisent en moyenne 9,5 $\pm 2,6$ heures de travail par jour. Le tableau I ci-dessous donne un aperçu généralde quelques caractéristiques sociodémographiques.

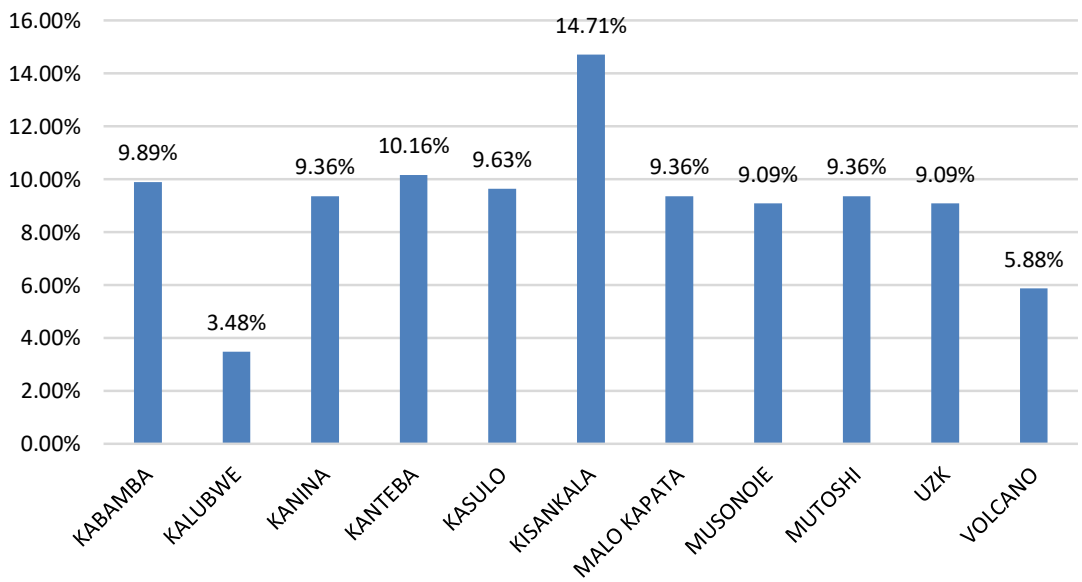

ont été retrouvées

Fig 1. Repartition des enquetées selon les sites ou elles

Tableau I: caractéristiques sociodémographiques des enquêtées

\begin{tabular}{|c|c|c|}
\hline Niveau d'étude $n=374$ & $\mathrm{Ni}$ & $\%$ \\
\hline Primaire & 189 & 50,53 \\
\hline Secondaire & 185 & 49,47 \\
\hline Etat matrimonial $n=374$ & $\mathrm{Ni}$ & $\%$ \\
\hline Célibataire & 42 & 11,23 \\
\hline Divorcé & 9 & 2,41 \\
\hline Marié & 303 & 81,02 \\
\hline Veuve & 20 & 5,35 \\
\hline Profession du mari $n=311$ & $\mathrm{Ni}$ & $\%$ \\
\hline Agriculteur & 12 & 3,86 \\
\hline Creuseur & 193 & 62,06 \\
\hline Négociant & 15 & 4,82 \\
\hline Sans emploi & 91 & 29,26 \\
\hline $\begin{array}{l}\text { Résidence par rapport aux sites } \\
\text { miniers } n=374\end{array}$ & $\mathrm{Ni}$ & $\%$ \\
\hline A proximité du site & 176 & 47,06 \\
\hline En dehors du site & 161 & 43,05 \\
\hline Intérieur du site & 37 & 9,89 \\
\hline Tranche d'âge $n=374$ & $\mathrm{Ni}$ & $\%$ \\
\hline$>10-20$ & 61 & 16,31 \\
\hline$>20-30$ & 164 & 43,85 \\
\hline$>30-40$ & 92 & 24,60 \\
\hline$>40-50$ & 44 & 11,76 \\
\hline$>50-60$ & 13 & 3,48 \\
\hline
\end{tabular}



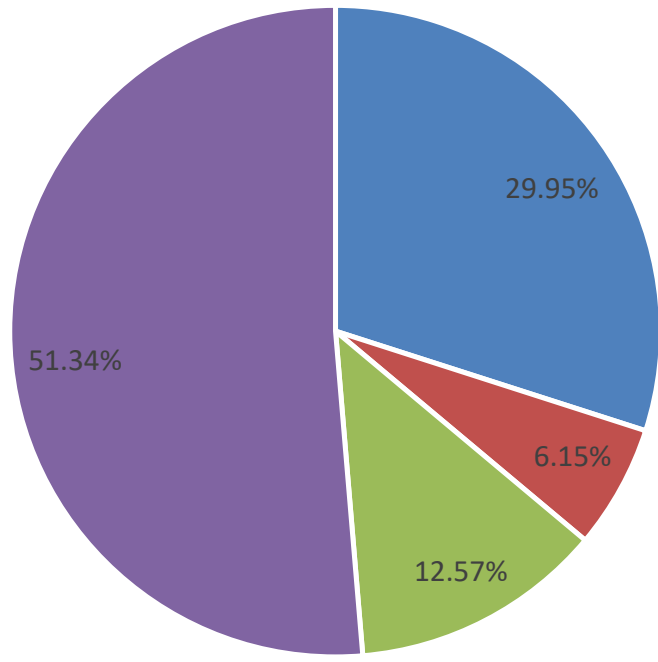

- Aider le conjoint

- Activités marchandes payantes

n Gain facile

- activité de survivance

Fig. 2 motivation des enquétées par rapport à leur presence dans les sites miniers

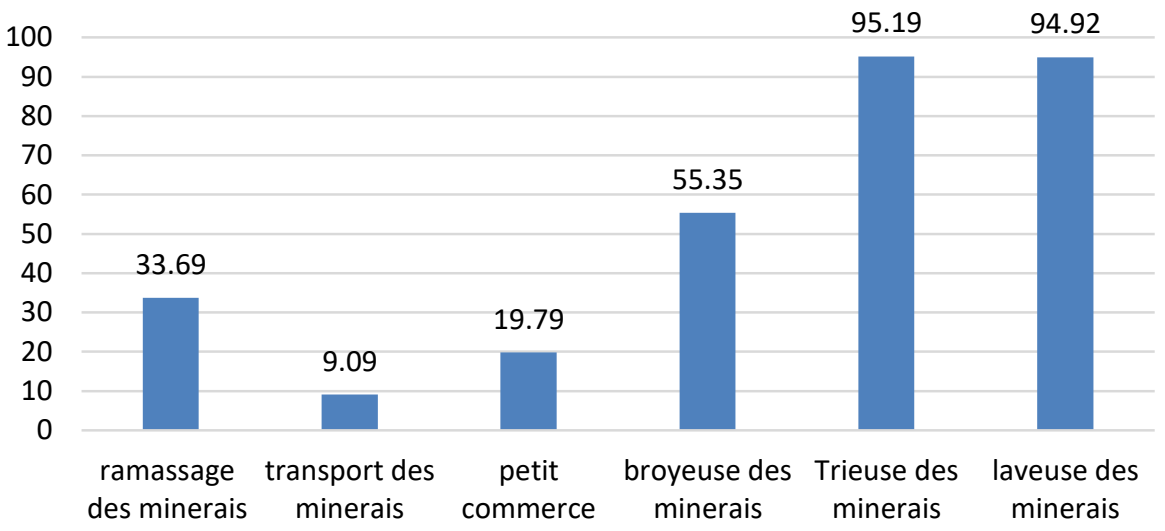

Fig. 3 activités prevalentes des enquétées dans les sites miniers

Tableau II : Répartition des résultats par rapport aux comportements à risque associés à l'activité minière observée auprès des femmes enquêtées

\begin{tabular}{ccc}
\hline Variables & ni & $\%$ \\
\hline Absence de port d'EPI & $349 / 374$ & 93,32 \\
Absence de port de Masque & $372 / 374$ & 99,47 \\
Absence de port de gant & $374 / 374$ & 100,00 \\
Absence de port de Casque & $372 / 374$ & 99,47 \\
Absence de port de bottes & $351 / 374$ & 93,85 \\
Absence de port de Lunette & $373 / 374$ & 99,73 \\
Entreposage des minerais dans la maison & $237 / 374$ & 63,37 \\
Lavage des parties du corps avec l'eau d'exhaure & $284 / 374$ & 75,94 \\
Station assise sur les minerais lors du travail & $255 / 374$ & 68,18 \\
Femmes trouvées travaillant avec les membres de la famille & $220 / 374$ & 58,82 \\
\hline
\end{tabular}

En ce qui concerne les raisons ayant poussées les enquêtées à travailler dans les sites miniers, ainsi que leur connaissance sur le risque inhérent aux minerais manipulés, nous avons observé qu'à peu près la moitié de l'échantillon soit $51,34 \%$ des femmes ont déclarées que leur présence dans ce secteur était motivée par le fait que la mine artisanale constituait une activité de survivance de leurs ménages et 6,15\% d'entre elles ont estimé qu'elles se retrouvé dans les sites miniers parce que leurs activités marchandes payent mieux dans ce secteur. Par ailleurs 93,7\% des femmes ont déjà entendu parler de risque d'irradiation à cause de la présence des produits uranifère dans ce secteur, mais dans $86,7 \%$ ce risque est sous-estimé. La figure 2 ci-dessous reparti les 67 | Int. J. of Multidisciplinary and Current research, Vol.6 (Jan/Feb 2018) 
enquêtées par rapport aux motivations de leur présence dans les sites miniers artisanaux.

Concernant les activités réalisées par les femmes dans les sites miniers, notre enquête nous a révélé que les activités des femmes dans ce secteur étaient dominées par le triage des minerais (95,19\%); suivi du lavage des minerais $(94,92 \%)$; et le broyage des minerais $(55,35 \%)$. Le ramassage, le transport et le petit commerce étaient les activités les moins réalisées par les femmes. La figure 3 ci-dessous montre les activités les plus prévalentes réalisées par les femmes dans ce secteur de la vie sociale.

Les résultats se rapportant au comportement à risque ont indiqué une prévalence de $93,32 \%$ des femmes qui travaillaient sans EPI; de manière spécifique $99,47 \%$ d'entre elles ne portaient pas des masques, $100 \%$ travaillaient mains nues sans gants ; $99,47 \%$ ne portaient pas des casques; $93,85 \%$ ne portaient pas des bottes; $99,73 \%$ ne portaient pas des lunettes ; le comportement consistant à entreposer les minerais dans la maison a été attesté par $63,37 \%$ des ouvrières, et la station assise sur les minerais pendant les opérations de broyage ou de triage a été observé chez $68,18 \%$ des ouvrières. Le tableau II ci-dessous décrit les différents comportements à risques observés.

Après stratification des données, nous avons observé que dans les sites de Kanina, Kanteba, Kasulo, et Volcano aucune femme n'a été trouvée avec un EPI, dans ces mêmes sites plus de $50 \%$ des femmes trouvées étaient assises sur les minerais lors des opérations de broyages et de triages. Le comportement consistant à entreposer les minerais dans la maison était plus attesté par les femmes rencontrées dans les sites de Kabamba, Kalumbwe, Kanina, Kisankala, Mutoshi, UZK, Musonoie et Volcano. Par ailleurs, dans tous les sites 64 à $91 \%$ des femmes ont attesté avoir utilisé l'eau d'exhaure pour assurer la propreté de certaines parties du corps. Le tableau III cidessous décrit le comportement à risques les plus prévalent après stratification par la variable site minier.

Tableau III : Répartition des comportements à risque associés à l'activité des ouvrières dans les sites miniers après stratification

\begin{tabular}{|c|c|c|c|c|c|c|c|c|}
\hline \multirow[t]{2}{*}{ Sites } & \multicolumn{2}{|c|}{$\begin{array}{l}\text { Entreposage des } \\
\text { minerais dans la } \\
\text { maison }\end{array}$} & \multicolumn{2}{|c|}{$\begin{array}{l}\text { Station assise sur les } \\
\text { minerais lors des } \\
\text { opérations }\end{array}$} & \multicolumn{2}{|c|}{ femmes sans EPI } & \multicolumn{2}{|c|}{$\begin{array}{c}\text { Utilisation de l'eau } \\
\text { d'exhaure pour lavage } \\
\text { des parties du corps }\end{array}$} \\
\hline & ni & $\%$ & ni & $\%$ & ni & $\%$ & ni & $\%$ \\
\hline Kabamba & 33 & 89,19 & 33 & 89,19 & 35 & 94,59 & 24 & 64,86 \\
\hline Kalubwe & 12 & 92,31 & 12 & 92,31 & 13 & 100,0 & 11 & 84,62 \\
\hline Kanina & 28 & 80,00 & 28 & 80,00 & 31 & 88,57 & 24 & 68,57 \\
\hline Kanteba & 08 & 21,05 & 08 & 21,05 & 38 & 100,0 & 28 & 73,68 \\
\hline Kasulo & 07 & 19,44 & 07 & 19,44 & 36 & 100,0 & 28 & 77,78 \\
\hline Kisankala & 48 & 87,27 & 48 & 87,27 & 54 & 98,18 & 46 & 83,64 \\
\hline Malo Kapata & 10 & 29,41 & 10 & 29,41 & 30 & 88,24 & 27 & 79,41 \\
\hline Musonoie & 18 & 52,94 & 18 & 52,94 & 30 & 88,24 & 31 & 91,18 \\
\hline Mutoshi & 25 & 71,43 & 25 & 71,43 & 28 & 80,00 & 31 & 88,57 \\
\hline UZK & 26 & 76,47 & 26 & 76,47 & 31 & 91,18 & 19 & 55,88 \\
\hline Volcano & 22 & 100,00 & 22 & 100,0 & 22 & 100,0 & 15 & 68,18 \\
\hline
\end{tabular}

\section{Discussion}

Les hommes comme les femmes participent tous à l'exploitation minière artisanale en tant qu'ouvriers à différentes échelles. Dans notre étude, nous avons observé que les femmes étaient affectés aux opérations de tris dans $95,2 \%$; et aux opérations de lavage dans 94,9\%; ces opérations étaient réalisées sans EPI dans 93,32\% des cas, et ce en dépit du fait que ces activités sont taxées de plus toxique [14]. Ces résultats corroborent donc ceux de Pact Congo [11] qui note l'inexistence des moyens de protection rudimentaires chez tous les artisans miniers et il en est de même des observations d'Elenge M.[15] qui rapporte les conditions de travail déplorables des artisans miniers de la mine de Ruashi à Lubumbashi dans la province du haut Katanga, caractérisées par l'absence de port d'EPI.

Notre étude a indiqué que $99,47 \%$ des femmes ne portaient pas des masques, lorsqu'on associe ce comportement à l'activité de broyage, il y a lieu de croire délibérément que, les femmes affectées à cette activité inhalent des minéraux contenus dans les particules fines de diamètre aérodynamique très faibles qui s'échappent du concassage de la gangue minéralisée. Le journal officiel [16] corrobore cette considération en arguant que la pénétration pulmonaire est tributaire de la taille des particules qui, lorsqu'elles sont inférieures à environ 5 microns, peuvent atteindre les alvéoles lorsque les ouvriers réalisent le travail sans protection des voies respiratoires alors que les plus grosses sont arrêtées dans l'arbre bronchique, recrachées ou ingurgitées. Cette littérature corrobore le caractère pernicieux de l'activité de broyage, dans la mesure où, la voie respiratoire est la plus prépondérante en termes de pénétration des toxiques dans l'exploitation minière artisanale [17].

En outre, notre étude a montré que $100 \%$ des femmes travaillaient mains nues sans gants et 93,85\% d'entre elles ne portaient pas des bottes dans les opérations de lavage et de triage qui généralement se font dans des cours d'eau ou lacs de retenus. La littérature note cependant que les mines abandonnées et les activités minières à Kolwezi, constituent d'importantes sources 
des métaux toxiques affectant les compartiments environnants [18] et la concentration de ces métaux dans les eaux des rivières qui constituent pour l'essentiel l'exhaure excède les normes de l'OMS et d'Aquatic Quality Guidelines for the Protection of Aquatic Life recommandations $[19,20]$. Une autre étude signale à ce sujet des risques d'exposition associée à la nature des activités menées dans un cours d'eau soumis à la pression anthropique [21]. Toutes ces acceptions en association avec les résultats observés sur notre échantillon font de l'absence de port des bottes, gants lors des opérations de lavage et de triage comme comportements et pratiques à risque, et confère à ces activités le caractère toxique [14]. A la situation évoquée, lorsqu'on applique le nombre d'heure de travail $(9,5 \pm 2$ heures) et la durée dans l'activité $(3,3 \pm 2,3$ ans), qui peuvent signifier le temps de contact de ces femmes avec les minerais radiotoxiques. Tout, porte à croire à une possible exposition chronique de ces femmes.

Il est donc judicieux de considérer à ce titre que nos enquêtées sont exposés aux risques sanitaires rapportés dans la littérature [22] qui note des risques d'intoxications mais aussi d'irradiation pouvant se révéler au niveau somatique par plusieurs problèmes sanitaires dans cette catégorie socio-professionnelle [11]. Ainsi donc l'hypothèse d'une possible influence de ce cadre pernicieux (activités minières artisanales) dans la survenue des certains problèmes de santé spécifiques aux femmes n'est pas à écarter.

Des études corroborent cet avis en trouvant des concentrations urinaires très élevées des métaux tel que $\mathrm{Cd}, \mathrm{Co}, \mathrm{Pb}$ et $\mathrm{U}$ respectivement $4,43,5$ et 4 fois plus élevées, chez les sujets des zones minières que chez les sujets témoins [23, 24]. D'autres recherches indiquent que les éléments radiotoxiques auxquelles ces femmes sont exposées, miment les œstrogènes naturels et peuvent être considérés à ce titre comme des xénohormones [25]; et perturbent la fonction endocrine [26]. D'autres études observent chez les femmes exposées aux irradiations des problèmes endocriniens, de troubles menstruels, des problèmes gynécologiques comparées à celles vivant dans des zones considérées saines $[27,28]$. Il est de même des troubles de la reproduction et endocriniens, de stérilité secondaire, des fausses couches, des malformations congénitales, des grossesses anembryonnaire, des troubles menstruels, des prématurités, et des naissances de nouveau-nés de faibles poids dans les populations associées à l'exploitation minière artisanale ou exposées à des métaux toxiques [10,11, 13, 29, 30].

Notre recherche a rapporté aussi que dans $63,37 \%$ le comportement consistant à entreposer les minerais dans la maison a été attesté par les femmes, et la station assise sur les minerais pendant les opérations de broyage ou de triage a été également observé chez 68,18\% d'entre elles. Et ce en dépit de la nature radiotoxique des minerais $[10,11,12,31,32]$. Une étude menée à Kapata corrobore ce résultat. En effet, les auteurs observent l'entreposage des produits miniers dans les maisons d'habitations et parfois les enfants jouent dessus indique cette source. Elle rapporte aussi des cas de malformations fœtales chez les mères parturientes qui, ayant exercé le lavage, et le triage des produits miniers artisanaux [33].

\section{Conclusion}

Au regard de tous les résultats, notre étude indique une possibilité d'exposition chronique des femmes aux minerais radiotoxiques, par le truchement du temps de contact avec les minerais qui est trop long, l'absence quasi-total des port des EPI, l'entreposage des minerais dans les maisons, la station assise sur les minerais au moment des opérations de broyage ou de triage, et l'utilisation des eaux d'exhaure pour assurer la propreté des certaines parties du corps et des ustensiles après le travail. Ces comportements exposent ces femmes aux risques d'irradiation et d'intoxication qui peuvent se révéler au niveau somatique par des troubles de plusieurs natures. Cependant, cette activité reste une de principales activités marchandes à impact économique essentiel pour les personnes ayant manqué l'emploi dans les industries du secteur privé. Le gouvernement à travers les ministères de mine, de santé publique et celui d'environnement et mines, devrait renforcer les mesures de protection et de sensibilisation à travers la cellule de santé au travail et de santé environnementale. A la société civile de faire des plaidoyers auprès du gouvernement et des organismes de droits humains en faveur de l'assainissement des conditions de travail de la femme dans ce secteur.

\section{References}

[1] Hinton, J., Veiga, M. M., \& Beinhoff, C. (2003), Women and artisanal mining: Gender roles and the road ahead. The socioeconomic impacts of artisanal and small-scale mining in developing countries, 336099-1163605893612.

[2]Amutabi, M. and Lutta-Mukhebi, M. (2001), Gender and Mining in Kenya: The Case of the Mukibira Mines in the Vihiga District, Jenda: A Journal of Culture and African Women Studies, Vol. 1, No. 2, 23p.

[3] Onuh, B. (2002). Salt Women of Keana, from. Newswatch.

[4] Blair, D., Rutherford, B., O'Neill, M., Vargas-Garcia, A., \& Melesse, M. (2017). Favoriser l'autonomisation des femmes dans l'exploitation minière artisanale et à petite échelle en Afrique centrale et en Afrique de l'Est.

[5] Keita, S. (2001). Étude sur les mines artisanales et les exploitations minières à petite échelle au Mali. International Institute for Environment and Development. London.

[6] Yakovleva, N. (2007). Perspectives on female participation in artisanal and small-scale mining: A case study of Birim North District of Ghana. Resources Policy, 32(1), 29-41.

[7] Rapport d'octobre 2007 de la banque mondiale intitulé : la bonne gouvernance dans le secteur minier comme facteur de croissance, octobre 2007.

[8] MONUSCO (2010), Rapport de l'étude sur l'impact de l'exploitation artisanale des ressources naturelles sur le 69 | Int. J. of Multidisciplinary and Current research, Vol.6 (Jan/Feb 2018) 
développement, l'autonomisation et l'avancement de la femme et de la fille en RDC. (nord-Kivu, Sud-kivu, Manièma-oriental, Kasai-occidental, Kasai-oriental et Katanga), Kinshasa ; p.14.

[9] Hayes, K., \& Perks, R. (2012). Women in the artisanal and small-scale mining sector of the Democratic Republic of the Congo. High-value Natural Resources and Post-Conflict Peacebuilding; Routledge: New York, NK, USA, 529-544.

[10] Pact Congo (2010), Coper, Cobalt and conflict: creating the capacity, mechanisms and relationships for reducing conflict in artisanal mining sector of Kolwezi, USAID DRC Mission, and Kinshasa; p. 22.

[11]Pact Congo. (2011), Health problems potentially linked to exposure to radioactive and toxic mineral substances in Kolwezi, copper, cobalt, and conflict in the artisanal mining sector of Kolwezi cooperative agreement, USAID Mision, Kinshasa; p. 27. [12] Lwamba llonda R., Mbweb Katshil P., Mwenge Kahinda J., and Tony Kayij Kanz Ntet (2017), Study of the Prevention of the Risks of Contamination and External Exposure of Persons Working in Mineral Crafts (Case of Musompo Site) International Journal of Innovation in Science and Mathematics Volume 5, Issue 4, ISSN (Online): 2347-9051

[13] Musao, J. K. (2009). La problématique de l'exploitation minière artisanale dans la province du Katanga (cas du district de Kolwezi). Mémoire Licence sociologie industrielle.

[14] Du Congo alimentent, I. C. M. (2015), Voilà pourquoi on meurt.

[15] Elenge Molayi, M., Aubry, J. C., \& De Brouwer, C. (2009). Impact des conditions de travail sur la santé des artisans miniers de la Ruashi (République démocratique du Congo). Médecine tropicale, 35(5), 488.

[16] Décret $n^{\circ} 038 / 2003$ du 26 mars 2003 portant règlement minier. In : Journal Officiel Numéro Spécial-1er avril 2003, Titre X, chapitre I, Article 230.

[17] Elenge Molayi, M., \& De Brouwer, C. (2010). Revue de la littérature des pathologies liées aux risques toxicologiques dans l'exploitation minière artisanale au Katanga (RDC). Journal International de Santé au Travail.

[18] Atibu, E. K., Lacroix, P., Sivalingam, P., Ray, N., Giuliani, G., Mulaji, C. K., ... \& Poté, J. (2018). High contamination in the areas surrounding abandoned mines and mining activities: An impact assessment of the Dilala, Luilu and Mpingiri Rivers, Democratic Republic of the Congo. Chemosphere, 191, 10081020.

[19] Atibu, E. K., Devarajan, N., Thevenon, F., Mwanamoki, P. M., Tshibanda, J. B., Mpiana, P. T \& Poté, J. (2013). Concentration of metals in surface water and sediment of Luilu and Musonoie Rivers, Kolwezi-Katanga, Democratic Republic of Congo. Applied geochemistry, 39, 26-32.

[20] Pourret, O., Lange, B., Bonhoure, J., Colinet, G., Decrée, S., Mahy, G., ... \& Faucon, M. P. (2016). Assessment of soil metal distribution and environmental impact of mining in Katanga (Democratic Republic of Congo). Applied Geochemistry, 64, 4355.
[21] Kalala, S. K., Mwanga, B. M., Kanyama, P. K., \& Mubemba, M. M. (2015). Impact des rejets miniers liquides de l'usine Chemical of Africa (CHEMAF) en activité sur la qualité des eaux souterraines au Quartier Tshamilemba à Lubumbashi (Katanga/RD Congo)[Impact of liquid tailings of Chemical Plant of Africa (CHEMAF) in activity on groundwater quality in the area Tshamilemba in Lubumbashi (Katanga/DR. Congo)].

[22] Elenge, M. M., Aubry, J. C., Jacob, L., \& De Brouwer, C. (2011). Heavy metal in hair samples of 109 non-industrial (miners) population in Katanga. Cahiers d'études et de recherches francophones/Santé, 21(1), 41-46.

[23] Banza, C. L. N., Nawrot, T. S., Haufroid, V., Decrée, S., De Putter, T., Smolders, E., ... \& Nemery, B. (2009). High human exposure to cobalt and other metals in Katanga, a mining area of the Democratic Republic of Congo. Environmental research, 109(6), 745-752.

[24] Nkulu, C. B. L., Nawrot, T., Haufroid, V., Lison, D., Smolders, E., \& Nemery, B. (2007). Biomonitoring of metals in the population of southern Katanga, a mining area of the DR Congo. Epidemiology, 18(5), S131-S132.

[25] Krishnan, A. V., Stathis, P., Permuth, S. F., Tokes, L., \& Feldman, D. (1993). Bisphenol-A: an estrogenic substance is released from polycarbonate flasks during autoclaving. Endocrinology, 132(6), 2279-2286.

[26] Bandajevsky Yu.I. (1999) Pathology of incorporated ionizing radiation. Gomel State Med. Inst. Minsk, 136. (In Russian).

[27] Nesterenko V.B., Yakovlev V.A., Nazarov A.G. (Eds.). (1993) Chernobyl accident. Reasons and consequencies (Expert conclusion). Part 4. Consequences for Ukraine and Russia. Minsk, "Test" Publ., 243 p. (in Russian).

[28]Kulakov V.I., Sokur A.L., Volobuev A.L. et al. (1993). Female reproductive functions in areas affected by radiation after the Chernobyl power station accident. Environ. Health Perspect. Suppl., Vol. 101

[29] Andrews, K. W., Savitz, D. A., \& Hertz-Picciotto, I. (1994). Prenatal lead exposure in relation to gestational age and birth weight: a review of epidemiologic studies. American journal of industrial medicine, 26(1), 13-32.

[30] Melis, B., Bocquene, G., \& Raffin, B. (1999). Effets endocriniens des contaminants en milieu marin.

[31] Makabu, K., Charlet, J. M., Doremus, P., \& Quinif, Y. (1990). Comportement des radioéléments dans les formations latéritiques des environs de Lubumbashi (Shaba, Zaïre), application à la prospection des gisements $\mathrm{Cu}-\mathrm{Co}-\mathrm{U}$. Annales de la Société géologique de Belgique.

[32] Charlet, J. M., \& MAKABU, K. (1996). Le radon en prospection minière, application à la recherche des minéralisations de la province cuprocobaltifère du Shaba (Congo). Bulletin de la Société belge de géologie, 105(1-2), 5-14.

[33] Mutshima, C. K., Kindele, J. M., Tshimwanga, J. S., \& Tshianzula, P. K. (2016), de l'exploitation minière artisanale et son impact environnemental dans la ville de Kolwezi. KAS African Law Study Library, 2(3), 581-597. 